\title{
The Core-EP, Weighted Core-EP Inverse of Matrices and Constrained Systems of Linear Equations
}

\author{
Jun $\mathrm{Ji}^{1, *}$ and Yimin $\mathrm{Wei}^{2}$ \\ ${ }^{1}$ Department of Mathematics, Kennesaw State University, \\ 1100 S. Marietta Pkwy, Marietta, GA 30060, USA. \\ ${ }^{2}$ School of Mathematical Sciences and Shanghai Key Laboratory \\ of Contemporary Applied Mathematics, Fudan University, \\ Shanghai 200433, China.
}

Received 28 August 2020; Accepted 14 October 2020

Dedicated to Prof. Jiaoxun Kuang's 85-th Birthday

\begin{abstract}
We study the constrained system of linear equations

$$
A x=b, \quad x \in \mathcal{R}\left(A^{k}\right)
$$

for $A \in \mathbb{C}^{n \times n}$ and $b \in \mathbb{C}^{n}, k=\operatorname{Ind}(A)$. When the system is consistent, it is well known that it has a unique $A^{D} b$. If the system is inconsistent, then we seek for the least squares solution of the problem and consider

$$
\min _{x \in \mathcal{R}\left(A^{k}\right)}\|b-A x\|_{2}
$$

where $\|\cdot\|_{2}$ is the 2-norm. For the inconsistent system with a matrix $A$ of index one, it was proved recently that the solution is $A^{\oplus} b$ using the core inverse $A^{\oplus}$ of $A$. For matrices of an arbitrary index and an arbitrary $b$, we show that the solution of the constrained system can be expressed as $A^{\oplus} b$ where $A^{\oplus}$ is the core-EP inverse of $A$. We establish two Cramer's rules for the inconsistent constrained least squares solution and develop several explicit expressions for the core-EP inverse of matrices of an arbitrary index. Using these expressions, two Cramer's rules and one Gaussian elimination method for computing the
\end{abstract}

\footnotetext{
*Corresponding author. Email addresses: j ji@kennesaw.edu (J. Ji), ymwei@fudan.edu.cn, yimin. wei@gmail.com (Y. Wei )
} 
core-EP inverse of matrices of an arbitrary index are proposed in this paper. We also consider the $W$-weighted core-EP inverse of a rectangular matrix and apply the weighted core-EP inverse to a more general constrained system of linear equations.

AMS subject classifications: 15A09, 15A24, 15A29, 15A57

Key words: Bott-Duffin inverse, Core-EP inverse, weighted core-EP inverse, Cramer's rule, Gaussian elimination method.

\section{Introduction}

Let $\mathbb{C}$ be the field of complex numbers and $\mathbb{C}^{m \times n}$ be the set of all $m \times n$ matrices over $\mathbb{C}$. For a matrix $A \in \mathbb{C}^{m \times n}, A^{T}, A^{*}, \mathcal{R}(A), \mathcal{N}(A)$, and $\operatorname{Ind}(A)$ stand for its transpose, conjugate transpose, range, null space, and index. $I$ is the identity matrix of order $n$ and $e_{i}$ is the $i$-th column of $I$. The Moore-Penrose inverse $A^{+}$of $A$ is the unique matrix $X \in \mathbb{C}^{n \times m}$ satisfying

$$
\begin{aligned}
& A X A=A, \\
& X A X=X, \\
& (A X)^{*}=A X, \\
& (X A)^{*}=X A .
\end{aligned}
$$

The matrix $X$ satisfying the 1st and 3rd matrix equations of the system of matrix equations (1.1)-(1.4) is called a $\{1,3\}$-inverse of $A$, denoted by $A^{(1,3)}$ and the collection of all $\{1,3\}$-inverses of $A$ is denoted by $A\{1,3\}$. It is well known that $A^{\dagger}=A^{-1}$ for a nonsingular square matrix $A$ and that $A^{\dagger} b$ is the minimum norm least squares solution of the system of linear equations $A x=b$ for a general matrix $A \in \mathbb{C}^{m \times n}$ and $b \in \mathbb{C}^{m}$.

The Drazin inverse $A^{D}$ of a square matrix $A \in \mathbb{C}^{n \times n}$ is the unique matrix $X \in$ $\mathbb{C}^{n \times n}$ satisfying

$$
X A X=X, \quad X A^{k+1}=A^{k}, \quad A X=X A
$$

for $k=\operatorname{Ind}(A)$. It is well known that both $A^{\dagger}$ and $A^{D}$ coincide with $A^{-1}$ for nonsingular matrices. For the special case when $\operatorname{Ind}(A)$ is one, the Drazin inverse is called the group inverse and is denoted by $A^{\#}$. The group inverse is a useful tool in the study of Markov chains and the Drazin inverse is used to study the singular differential and difference equations [7]. It is well known that the constrained system of linear equations

$$
A x=b, \quad x \in \mathcal{R}\left(A^{k}\right)
$$


for $k=\operatorname{Ind}(A)$, is consistent if and only if $b \in \mathcal{R}\left(A^{k}\right)$ and the unique solution is given by $A^{D} b$ if it is consistent [5, p. 167]. The result has been extended to the tensor case [19].

When the constrained system of linear equations (1.6) is inconsistent, one would naturally consider the least squares solution of the system and seeks to solve the following constrained optimization problem:

$$
\min _{x \in \mathcal{R}\left(A^{k}\right)}\|b-A x\|_{2}
$$

where $\|\cdot\|_{2}$ is the 2-norm in $\mathbb{C}^{n}$.

It is shown that for $A \in \mathbb{C}_{n}^{C M}$, where

$$
\mathbb{C}_{n}^{\mathrm{CM}}=\left\{A: \operatorname{Ind}(A) \leq 1, A \in \mathbb{C}^{n \times n}\right\},
$$

the constrained least squares problem (1.7) has a unique solution $A^{\oplus} b[23,26,46]$ where $A^{\oplus}$, called the core inverse of $A$, is the unique matrix $X \in \mathbb{C}^{n \times n}$ satisfying

$$
A X=A A^{\dagger}, \quad \mathcal{R}(X) \subseteq \mathcal{R}(A) .
$$

It was pointed out that the core inverse of $A$ was introduced in [2] as an alternative to the group inverse as it relates closely to the group inverse

$$
A^{\oplus}=A^{\#} A A^{\dagger}
$$

and that it is indeed the Bott-Duffin inverse of $A$ with respect to $\mathcal{R}(A)$

$$
A^{\oplus}=A_{(\mathcal{R}(A))}^{(-1)}=A A^{\dagger}\left[I+(A-I) A A^{\dagger}\right]^{-1} .
$$

Since the birth of the core inverse of matrices of index one, researchers in the area have shown great interest in the generalized inverse and have obtained many algebraic and geometric properties of the inverse and designed Cramer's rules, Gauss elimination methods, and the neural network for its computation $[2,20,21$, $23,28,31,37,42-44,46,48]$.

The core inverse of a matrix of index one has been extended to matrices of an arbitrary index in several different ways in the literature. The one proposed in [30] is the unique matrix $X$ satisfying

$$
X A X=X, \quad(A X)^{*}=A X, \quad X A^{l+1}=A^{l}, \quad \mathcal{R}(X) \subseteq \mathcal{R}\left(A^{l}\right)
$$

for $l \geq \operatorname{Ind}(A)$. This generalized inverse is called the core-EP inverse of $A$ and is denoted by $A^{\oplus}$. It is actually the $\{2\}$-inverse of $A$ with prescribed range $\mathcal{R}\left(A^{k}\right)$ and nullspace $\mathcal{N}\left(\left(A^{k}\right)^{*}\right)$

$$
A^{\oplus}=A_{\mathcal{R}\left(A^{k}\right), \mathcal{N}}^{(2)}\left(\left(A^{k}\right)^{*}\right)^{.}
$$


We remark that the paper [30] actually defines the core-EP inverse of matrices over a general field. It may not exist over a general field but it always exists and is unique for the matrices over $\mathbb{C}$ and according to [30, Theorem 3.5, Remark 2],

$$
A^{\oplus}=A^{k}\left(\left(A^{*}\right)^{k} A^{k+1}\right)^{\dagger}\left(A^{*}\right)^{k}
$$

for $k=\operatorname{Ind}(A)$. In addition to the ones in (1.12) and (1.13), we have the following useful explicit expressions for the core-EP inverse $[27,40]$

$$
A^{\oplus}=A^{D} A^{k}\left(A^{k}\right)^{\dagger}=A^{k}\left(A^{k+1}\right)^{\oplus}=A^{k}\left(A^{k+1}\right)^{\dagger}, \quad k=\operatorname{Ind}(A)
$$

as well as a full rank decomposition-based expression and several numerical methods for the core-EP inverse in the literature [27,31,32,47].

The other extensions of the core inverse of $A$ from $\mathbb{C}_{n}^{\mathrm{CM}}$ to $\mathbb{C}^{n \times n}$ include the generalized core inverse $A^{\diamond}[3]$, the DMP inverse $A^{d, \dagger}[25,29]$, and the weak group inverse [41]. The generalized core inverse $A^{\diamond}$ and the DMP inverse $A^{d, \dagger}$ can be expressed as

$$
A^{\diamond}=\left(A P_{A}\right)^{\dagger}=A A^{\dagger}\left[I+(A-I) A A^{\dagger}\right]^{\dagger}, \quad A^{d,+}=A^{D} A A^{\dagger} .
$$

When $A \in \mathbb{C}_{n}^{\mathrm{CM}}$, we have

$$
A^{\oplus}=A^{\diamond}=A^{d, \dagger}=A^{\oplus}
$$

due to (1.9), (1.10), (1.15), (1.14), and the facts that $I+(A-I) A A^{\dagger}$ is nonsingular and that $A^{D}=A^{\#}$ for matrices of index one.

For the weak group inverse of $A$ in $\mathbb{C}^{n \times n}$, the pseudo core inverse in ring with involution, and the core inverse of Hilbert space operators, please refer to $[12,33,41]$.

In this paper we further investigate the constrained optimization problem (1.7). We show that the solution can be expressed as $A^{\oplus} b$. Two Cramer's rules for the least squares solution $A^{\oplus} b$ to (1.7) and two determinantal formulas and one Gaussian elimination method for computing the core-EP inverse $A{ }^{\oplus}$ of $A$ are developed. Several other explicit expressions for the core-EP inverse will also be established, extending the existing results for the core inverse in the literature to the core-EP inverse. Finally, we extend a result for core-EP inverse of a square matrix to the weighted core-EP inverse of a rectangular matrix and apply it to a more general constrained system of linear equations. 


\section{A constrained system of linear equations}

We begin with a more general constrained system of linear equations considered in [9]:

$$
A x=b, \quad x \in T,
$$

where $T$ is a subspace of $\mathbb{C}^{n}$ and when (2.1) is inconsistent, consider

$$
\min _{x \in T}\|b-A x\|_{2}
$$

The coefficient matrix $A$ in (2.1) and (2.2) is allowed to be rectangular in the original setting but we still assume that it is square here. It is shown in [9, Theorem 3.4] that both (2.1) and (2.2) have a unique solution if and only if $T \cap \mathcal{N}(A)=\{0\}$ and the unique solution is given by $x=\left(A^{*} A\right)_{(T)}^{(-1)} A^{*} b$. We notice that the problems (1.6) and (1.7) are special cases of the aforementioned problems when $T=\mathcal{R}\left(A^{k}\right)$. Due to the fact that

$$
T \cap \mathcal{N}(A) \subseteq \mathcal{R}\left(A^{k}\right) \cap \mathcal{N}\left(A^{k}\right)=\{0\},
$$

we immediately have the following result.

Observe that having a zero minimum value of (2.2) is equivalent to having a consistent system (2.1). Thus, (2.1) is contained in (2.2) as a special case when the minimum value is zero.

Theorem 2.1. Let $A \in \mathbb{C}^{n \times n}, b \in \mathbb{C}^{n}$, and $k=\operatorname{Ind}(A)$ The problem (1.7) has a unique solution

$$
x=\left(A^{*} A\right)_{\left(\mathcal{R}\left(A^{k}\right)\right)}^{(-1)} A^{*} b=A^{k}\left(A^{k}\right)^{\dagger}\left[I+\left(A^{*} A-I\right) A^{k}\left(A^{k}\right)^{\dagger}\right]^{-1} A^{*} b .
$$

Proof. We only need to show that the matrix involved in the Bott-Duffin inverse is nonsingular. Assume that

$$
\left[I+\left(A^{*} A-I\right) A^{k}\left(A^{k}\right)^{\dagger}\right] x=0 .
$$

Then we have

$$
x=\left(I-A^{*} A\right) A^{k}\left(A^{k}\right)^{\dagger} x
$$

implying that

$$
\left(A^{k}\right)^{\dagger} x=\left(A^{k}\right)^{\dagger}\left(I-A^{*} A\right) A^{k}\left(A^{k}\right)^{\dagger} x=\left(A^{k}\right)^{\dagger} x-\left(A^{k}\right)^{\dagger} A^{*} A A^{k}\left(A^{k}\right)^{\dagger} x,
$$


which leads to $\left(A^{k}\right)^{\dagger} A^{*} A A^{k}\left(A^{k}\right)^{\dagger} x=0$. Now, we have

$$
\begin{aligned}
\left\|A A^{k}\left(A^{k}\right)^{\dagger} x\right\|_{2}^{2} & =\left(A A^{k}\left(A^{k}\right)^{\dagger} x\right)^{*} A A^{k}\left(A^{k}\right)^{\dagger} x \\
& =x^{*} A^{k}\left(\left(A^{k}\right)^{\dagger} A^{*} A A^{k}\left(A^{k}\right)^{\dagger} x\right)=0
\end{aligned}
$$

leading to $A^{k}\left(A^{k}\right)^{\dagger} x \in \mathcal{N}(A) \subseteq \mathcal{N}\left(A^{k}\right)$. Therefore, $A^{k}\left(A^{k}\right)^{\dagger} x \in \mathcal{N}\left(A^{k}\right) \cap \mathcal{R}\left(A^{k}\right)=\{0\}$ which, together with (2.4), finally implies that $x=0$ and thus $I+\left(A^{*} A-I\right) A^{k}\left(A^{k}\right)^{\dagger}$ is nonsingular. The formula (2.3) follows directly from the expression for BottDuffin inverse [5, Sec. 10].

For $A \in \mathbb{C}_{n}^{\mathrm{CM}}$ and an arbitrary $b$, due to the fact the unique solution of constrained systems (1.6) and (1.7) is $A^{\oplus} b[23,26,46]$, we have

$$
A^{\oplus}=A A^{\dagger}\left[I+\left(A^{*} A-I\right) A A^{\dagger}\right]^{-1} A^{*},
$$

which is different from (1.10). As indicated by the following example, the difference is real. For the matrix $A \in \mathbb{C}_{n}^{\mathrm{CM}}$ given by [2]

$$
A=\left(\begin{array}{ll}
1 & 1 \\
0 & 0
\end{array}\right)
$$

we have

$$
A^{\dagger}=\left(\begin{array}{cc}
\frac{1}{2} & 0 \\
\frac{1}{2} & 0
\end{array}\right), \quad A^{*} A=\left(\begin{array}{cc}
1 & 1 \\
1 & 1
\end{array}\right), \quad A A^{\dagger}=\left(\begin{array}{ll}
1 & 0 \\
0 & 0
\end{array}\right)
$$

leading to

$$
\begin{aligned}
& {\left[I+\left(A^{*} A-I\right) A A^{\dagger}\right]^{-1} A^{*}=\left(\begin{array}{ll}
1 & 0 \\
0 & 0
\end{array}\right),} \\
& {\left[I+(A-I) A A^{\dagger}\right]^{-1}=I .}
\end{aligned}
$$

As expected, both formulas (2.5) and (1.10) give

$$
A^{\oplus}=\left(\begin{array}{ll}
1 & 0 \\
0 & 0
\end{array}\right)
$$

but the first one of (2.6) involves two more matrix multiplications. In general, the expression (2.5) is always computationally more intensive since it requires two more matrix multiplications for computing the core-EP inverse than does the expression (1.10). 
We feel that working with a general setting, the special structure of our problems is not explored in [9] at all. It is our goal to fully explore its special structure and get a result which could reduce to (1.10) at a minimum.

To this end, we begin with a technical result.

Lemma 2.1. Let $A \in \mathbb{C}^{n \times n}$ and $k=\operatorname{Ind}(A)$. Any square matrix $Y \in \mathbb{C}^{n \times n}$ satisfying $\mathcal{R}(Y) \subseteq \mathcal{R}\left(A^{k}\right) \cap \mathcal{N}(A)$ must be the zero matrix.

Proof. Let $x$ be an arbitrary vector in $\mathbb{C}^{n}$. We can write

$$
Y x \in \mathcal{R}(Y) \subseteq \mathcal{R}\left(A^{k}\right) \cap \mathcal{N}(A) \subseteq \mathcal{R}\left(A^{k}\right) \cap \mathcal{N}\left(A^{k}\right)=\{0\}
$$

implying $Y x=0$ for any $x$. Thus, we have $Y=O$.

It is well known [5, pp.166-167] that $A^{D}=A^{k}\left(A^{k+1}\right)^{\#}$ for $k=\operatorname{Ind}(A)$. The two expressions for the core-EP inverse in (1.14) have a similar nature. The following result characterizes the core-EP inverse.

Theorem 2.2. Let $A \in \mathbb{C}^{n \times n}$ and $l \geq k=\operatorname{Ind}(A)$. Then, $A^{l} Y$ is the core-EP inverse of $A$ if and only if $Y$ is a $\{1,3\}$-inverse of $A^{l+1}$.

Proof. Let $X=A^{l}\left(A^{l+1}\right)^{(1,3)}$. Obviously, $\mathcal{R}(X) \subseteq \mathcal{R}\left(A^{l}\right)=\mathcal{R}\left(A^{k}\right)$ and $A X=A^{l+1}$ $\left(A^{l+1}\right)^{(1,3)}$ implying $(A X)^{*}=A X$. A simple calculation leads to

$$
\begin{aligned}
& A(X A X-X) \\
= & A X A X-A X=A^{l+1}\left(A^{l+1}\right)^{(1,3)} A^{l+1}\left(A^{l+1}\right)^{(1,3)}-A^{l+1}\left(A^{l+1}\right)^{(1,3)}=O, \\
& A\left(X A^{l+1}-A^{l}\right)=A X A^{l+1}-A^{l+1}=A^{l+1}\left(A^{l+1}\right)^{(1,3)} A^{l+1}-A^{l+1}=O
\end{aligned}
$$

implying that

$$
\begin{aligned}
& \mathcal{R}(X A X-X) \subseteq \mathcal{N}(A), \\
& \mathcal{R}\left(X A^{l+1}-A^{l}\right) \subseteq \mathcal{N}(A) .
\end{aligned}
$$

In addition, we have

$$
\begin{aligned}
& X A X-X=A^{l}\left(\left(A^{l+1}\right)^{(1,3)} A X-\left(A^{l+1}\right)^{(1,3)}\right), \\
& X A^{l+1}-A^{l}=A^{l}\left(\left(A^{l+1}\right)^{(1,3)} A^{l+1}-I\right)
\end{aligned}
$$

implying that

$$
\mathcal{R}(X A X-X) \subseteq \mathcal{R}\left(A^{l}\right)=\mathcal{R}\left(A^{k}\right)
$$




$$
\mathcal{R}\left(X A^{l+1}-A^{l}\right) \subseteq \mathcal{R}\left(A^{l}\right)=\mathcal{R}\left(A^{k}\right)
$$

It follows from Lemma 2.1 that $X A X=X$ and $X A^{l+1}=A^{l}$. Therefore, all four conditions of (1.11) are verified. The uniqueness of the core EP inverse implies that $A^{\oplus}=A^{l}\left(A^{l+1}\right)^{(1,3)}$.

Now, assume that $A^{l} Y$ is the core-EP inverse of $A$. Then, $A^{\oplus}=A^{l} Y$ must satisfy all the conditions of (1.11). We can write

$$
A^{l+1} Y A^{l+1}=A\left(A^{l} \Upsilon\right) A^{l+1}=A\left(A^{\oplus} A^{l+1}\right)=A A^{l}=A^{l+1}
$$

implying $Y \in A^{l+1}\{1\}$. We also have

$$
A^{l+1} Y=A\left(A^{l} Y\right)=A A^{\oplus}
$$

implying that

$$
\left(A^{l+1} \Upsilon\right)^{*}=\left(A A^{\oplus}\right)^{*}=A A^{\oplus}=A^{l+1} \Upsilon .
$$

So, we also have $Y \in A^{l+1}\{3\}$.

For a general vector $b$, the constrained condition $x \in \mathcal{R}\left(A^{k}\right)$ of (1.7) can be removed by setting $x=A^{k} y$. Solving the problem (1.7) is equivalent to solving the following unconstrained least squares problem

$$
\min _{y \in \mathbb{C}^{n}}\left\|b-A^{k+1} y\right\|_{2}
$$

with $x=A^{k} y$. The general least squares solution of $A^{k+1} y=b$ is

$$
y=\left(A^{k+1}\right)^{(1,3)} b+\left(I-\left(A^{k+1}\right)^{(1,3)} A^{k+1}\right) q
$$

with $\left(A^{k+1}\right)^{(1,3)} \in A^{k+1}\{1,3\}$ and arbitrary $q[5$, Ch. 3, Sec. 1]. Thus, in view of Theorem 2.2, the general least squares solution of (1.7) is

$$
\begin{aligned}
x & =A^{k} y=A^{k}\left(\left(A^{k+1}\right)^{(1,3)} b+\left(I-\left(A^{k+1}\right)^{(1,3)} A^{k+1}\right) q\right) \\
& =A^{k}\left(A^{k+1}\right)^{(1,3)} b+A^{k}\left(I-\left(A^{k+1}\right)^{(1,3)} A^{k+1}\right) q \\
& =A^{\oplus} b+p,
\end{aligned}
$$

where

$$
p=A^{k}\left(I-\left(A^{k+1}\right)^{(1,3)} A^{k+1}\right) q .
$$


Observe that $p \in \mathcal{R}\left(A^{k}\right)$ and that

$$
\begin{aligned}
A p & =A A^{k}\left(I-\left(A^{k+1}\right)^{(1,3)} A^{k+1}\right) q \\
& =\left(A^{k+1}-A^{k+1}\left(A^{k+1}\right)^{(1,3)} A^{k+1}\right) q=0
\end{aligned}
$$

implying that $p \in \mathcal{N}(A) \subseteq \mathcal{N}\left(A^{k}\right)$. Thus,

$$
p \in \mathcal{R}\left(A^{k}\right) \cap \mathcal{N}(A) \subseteq \mathcal{R}\left(A^{k}\right) \cap \mathcal{N}\left(A^{k}\right)=\{0\}
$$

Therefore, we have $p=0$ and thus, the problem (1.7) has the unique solution $x=A^{\oplus} b$. In summary, we have proved the following result.

Theorem 2.3. Let $A \in \mathbb{C}^{n \times n}$ of an arbitrary index and any $b \in \mathbb{C}^{n}$. The problem (1.7) has a unique solution $A^{\oplus} b$.

Theorem 2.3 reduces to [46, Theorem 3.1] when $A$ is of index one. In view of Theorem 2.3, the core-EP inverse $A^{\oplus}$ of $A$ stands out among many generalizations of the core inverse of $A$ as we can express the least squares solution of (1.7) as $A^{\oplus} b$. Due to the importance of the Cramer's rule, we will develop two such rules for the least squares solution of (1.7) through two explicit expressions of the core-EP inverse in terms of nonsingular matrices in the next two sections. Several other explicit expressions for $A^{\oplus}$ will also be presented.

\section{Explicit expressions, QR-based and the Gaussian elimination methods for core-EP inverse}

Let us re-examine (1.7). Define $z=b-A x$ and in view of (2.8), we can write

$$
\begin{aligned}
z & =b-A x=b-A^{k+1} y \\
& =b-A^{k+1}\left(A^{k+1}\right)^{(1,3)} b-A^{k+1}\left(I-\left(A^{k+1}\right)^{(1,3)} A^{k+1}\right) q \\
& =b-A^{k+1}\left(A^{k+1}\right)^{(1,3)} b-\left(I-A^{k+1}\left(A^{k+1}\right)^{(1,3)}\right) A^{k+1} q \\
& =\left(I-A^{k+1}\left(A^{k+1}\right)^{(1,3)}\right)\left(b-A^{k+1} q\right) \\
& =\left(I-P_{\mathcal{R}\left(A^{k+1}\right)}\right)\left(b-A^{k+1} q\right)=\left(P_{\mathcal{R}\left(A^{k}\right)^{\perp}}\right)\left(b-A^{k+1} q\right) .
\end{aligned}
$$


Thus, $x$ solves (1.7) if and only if there exist $x$ in $\mathcal{R}\left(A^{k}\right)$ and $z \in \mathcal{R}\left(A^{k}\right)^{\perp}$ such that $A x+z=b$. Hence, (1.7) is equivalent to the Bott-Duffin system $[5,45]$

$$
A x+z=b, \quad x \in L, \quad z \in L^{\perp},
$$

where $L=\mathcal{R}\left(A^{k}\right)$ and $k=\operatorname{Ind}(A)$. For this special Bott-Duffin system, we have

$$
\begin{aligned}
& P_{L}=A^{k}\left(A^{k}\right)^{\dagger}, \quad P_{L^{\perp}}=I-A^{k}\left(A^{k}\right)^{\dagger}, \\
& A P_{L}+P_{L^{\perp}}=A^{k+1}\left(A^{k}\right)^{\dagger}+I-A^{k}\left(A^{k}\right)^{\dagger} .
\end{aligned}
$$

We can easily verify that $A P_{L}+P_{L^{\perp}}$ is always nonsingular. To this end, we assume that

$$
\left(A^{k+1}\left(A^{k}\right)^{\dagger}+I-A^{k}\left(A^{k}\right)^{\dagger}\right) w=0
$$

for some $w$, in view of (3.2). Thus, we have

$$
w=(I-A) A^{k}\left(A^{k}\right)^{\dagger} w=A^{k}(I-A)\left(A^{k}\right)^{\dagger} w \in \mathcal{R}\left(A^{k}\right) .
$$

For simplicity, let $w=A^{k} y$ for some $y$. Using (3.3) again, we have

$$
w=(I-A) A^{k}\left(A^{k}\right)^{\dagger} w=(I-A) A^{k}\left(A^{k}\right)^{\dagger} A^{k} y=(I-A) A^{k} y=(I-A) w
$$

implying $A w=0$. Thus, $w \in \mathcal{R}\left(A^{k}\right) \cap \mathcal{N}\left(A^{k}\right)=\{0\}$, i.e., $w=0$. Hence, $A P_{L}+P_{L^{\perp}}$ is nonsingular. According to the theory of the Butt-Duffin inverses [5,45], (3.1) has a unique solution

$$
x=P_{L}\left(A P_{L}+P_{L^{\perp}}\right)^{-1} b=A^{k}\left(A^{k}\right)^{\dagger}\left[I+(A-I) A^{k}\left(A^{k}\right)^{\dagger}\right]^{-1} b, \quad z=b-A x
$$

for any $b \in \mathbb{C}^{n}$ and $A^{k}\left(A^{k}\right)^{\dagger}\left[I+(A-I) A^{k}\left(A^{k}\right)^{\dagger}\right]^{-1}$ is the Bott-Duffin inverse $A_{\left(\mathcal{R}\left(A^{k}\right)\right)}^{(-1)}$ of $A$ with respect to $\mathcal{R}\left(A^{k}\right)$. In view of Theorem 2.3 and the equivalency of (1.7) and (3.1), we conclude that the core-EP inverse of $A$ is indeed the Bott-Duffin inverse of $A$ with respect to $\mathcal{R}\left(A^{k}\right)$ (refer to [5, Ch. 2, Sec. 10]).

In summary, we have proved the following result.

Theorem 3.1. Let $A \in \mathbb{C}^{n \times n}$ and $k=\operatorname{Ind}(A)$. Then $I+(A-I) A^{k}\left(A^{k}\right)^{\dagger}$ is nonsingular and

$$
A^{\oplus}=A_{\left(\mathcal{R}\left(A^{k}\right)\right)}^{(-1)}=A^{k}\left(A^{k}\right)^{\dagger}\left[I+(A-I) A^{k}\left(A^{k}\right)^{\dagger}\right]^{-1} .
$$


In view of Theorems 2.1 and 2.3, we have an alternative expression for the core-EP inverse of $A$

$$
A^{\oplus}=\left(A^{*} A\right)_{\left(R\left(A^{k}\right)\right)}^{(-1)} A^{*}=A^{k}\left(A^{k}\right)^{\dagger}\left[I+\left(A^{*} A-I\right) A^{k}\left(A^{k}\right)^{\dagger}\right]^{-1} A^{*} .
$$

This expression involves two more matrix multiplications than does the one in Theorem 3.1. More importantly, the formula in Theorem 3.1 does reduce to the one in (1.10).

Applying existing results for the Bott-Duffin inverse of $A$ with respect to $L=$ $\mathcal{R}\left(A^{k}\right)$, many new results can be obtained for the core-EP inverse. In particular, we would like to mention the one using the basis of the subspace $\mathcal{R}\left(A^{k}\right)$ (see [5, Sec. 10] or [45]).

Lemma 3.1 ([6]). Let $U$ be a matrix whose columns form a basis for L. Then the BottDuffin inverse $A_{(L)}^{(-1)}$ of $A$ exists if and only if $U^{*} A U$ is nonsingular, in which case,

$$
A_{(L)}^{(-1)}=U\left(U^{*} A U\right)^{-1} U^{*}
$$

Theorem 3.2. Let $A \in \mathbb{C}^{n \times n}$ and $k=\operatorname{Ind}(A)$. Let $U_{l}$ be a matrix whose columns form a basis for $\mathcal{R}\left(A^{l}\right)$. Then, $k$ is the smallest nonnegative integer $l$ such that $U_{l}^{*} A U_{l}$ being nonsingular and

$$
A^{\oplus}=U_{k}\left(U_{k}^{*} A U_{k}\right)^{-1} U_{k}^{*}
$$

Proof. Let $r_{l}=\operatorname{dim}\left(\mathcal{R}\left(A^{l}\right)\right)$. Then $\mathcal{R}\left(U_{l}\right)=\mathcal{R}\left(A^{l}\right)$ and $U_{l} \in \mathbb{C}_{r_{l}}^{n \times r_{l}}$. We also have

$$
\mathcal{R}\left(A^{l+1}\right)=A \mathcal{R}\left(A^{l}\right)=A \mathcal{R}\left(U_{l}\right)=\mathcal{R}\left(A U_{l}\right) .
$$

Obviously, $\mathcal{R}\left(A^{l+1}\right)=\mathcal{R}\left(A^{l}\right)$ is equivalent to $\mathcal{R}\left(A U_{l}\right)=\mathcal{R}\left(U_{l}\right)$, i.e., $A U_{l} \in \mathbb{C}_{r_{l}}^{n \times r_{l}}$.

If $\mathcal{R}\left(A^{l+1}\right)=\mathcal{R}\left(A^{l}\right)$, then $l \geq k$ and $\mathcal{R}\left(A U_{l}\right)=\mathcal{R}\left(U_{l}\right)$. Assume that $U_{l}^{*} A U_{l} x=0$ for any $x \in \mathbb{C}^{r_{l}}$. We have

$$
A U_{l} x \in \mathcal{N}\left(U_{l}^{*}\right)=\mathcal{R}\left(U_{l}\right)^{\perp} .
$$

On the other hand, $A U_{l} x \in \mathcal{R}\left(A U_{l}\right)=\mathcal{R}\left(U_{l}\right)$. Thus, we have

$$
A U_{l} x \in \mathcal{R}\left(U_{l}\right) \cap \mathcal{R}\left(U_{l}\right)^{\perp}=\{0\}
$$

implying that $A U_{l} x=0$. Since $U_{l} x \in \mathcal{N}(A) \subseteq \mathcal{N}\left(A^{l}\right)$, we have

$$
U_{l} x \in \mathcal{N}\left(A^{l}\right) \cap \mathcal{R}\left(A^{l}\right)=\{0\}
$$


which further implies $x=0$ due to the fact that the columns of $U_{l}$ are linearly independent. Therefore, $U_{l}^{*} A U_{l}$ is nonsingular.

Assume that $U_{l}^{*} A U_{l}$ is nonsingular. Then

$$
r_{l}=\operatorname{rank}\left(U_{l}^{*} A U_{l}\right) \leq \operatorname{rank}\left(A U_{l}\right) \leq r_{l}
$$

implying that $\operatorname{rank}\left(A U_{l}\right)=\operatorname{rank}\left(U_{l}\right)=r_{l}$. Thus, we have $\mathcal{R}\left(A U_{l}\right)=\mathcal{R}\left(U_{l}\right)$ which is equivalent to $\mathcal{R}\left(A^{l+1}\right)=\mathcal{R}\left(A^{l}\right)$.

We have just proved that $\mathcal{R}\left(A^{l+1}\right)=\mathcal{R}\left(A^{l}\right)$ is equivalent to the fact that $U_{l}^{*} A U_{l}$ is nonsingular. It follows from the definition of index that $k$ is the smallest nonnegative integer $l$ such that $U_{l}^{*} A U_{l}$ being nonsingular. Finally, the expression of the core-EP inverse directly follows from Lemma 3.1 and Theorem 3.1.

The expressions for various common generalized inverses of matrices based on the QR decomposition can be found in [38]. For the core-EP inverse, we have the following results.

Corollary 3.1. Let $A \in \mathbb{C}^{n \times n}$ and $k=\operatorname{Ind}(A)$.

1. If $A^{k}=P Q$ is a full rank decomposition of $A^{k}$, then

$$
A^{\oplus}=P\left(P^{*} A P\right)^{-1} P^{*} .
$$

2. Let $s=\operatorname{rank}\left(A^{k}\right)$ and let $A^{k}=Q R$ be the $Q R$ decomposition of $A^{k}$. If $Q_{1}$ is the first $s$ columns of $Q$, then

$$
A^{\oplus}=Q_{1}\left(Q_{1}^{*} A Q_{1}\right)^{-1} Q_{1}^{*}
$$

Proof. The first part of the corollary immediately follows from Theorem 3.2 and the fact that the columns of $P$ form a basis for $\mathcal{R}\left(A^{k}\right)$. For the second part, we assume that $R_{1}$ is the first $s$ rows of $R$. Then, we have $A^{k}=Q R=Q_{1} R_{1}$ which is also a full rank decomposition of $A^{k}$. Thus, the columns of $Q_{1}$ form a basis for $\mathcal{R}\left(A^{k}\right)$ and the result directly follows from Theorem 3.2.

We remark that an expression for the core-EP inverse of $A$ from a full rank decomposition is given in [27, Theorem 2.4]

$$
A^{\oplus}=P(Q A P)^{-1} Q P\left(P^{*} P\right)^{-1} P^{*} .
$$

Obviously, the expression (3.5) for the core-EP inverse of $A$ is much simpler and it involves much less work for computing the core-EP inverse. 
When $A$ is of index one, we have $A=P Q$ and in this case, the expression (3.5) can be written as

$$
A^{\oplus}=A^{\oplus}=P\left(P^{*}(P Q) P\right)^{-1} P^{*}=P(Q P)^{-1}\left(P^{*} P\right)^{-1} P^{*}
$$

since both $P^{*} P$ and $Q P$ are nonsingular. Thus, (3.5) reduces to the formula for $A^{\oplus}\left[43\right.$, Theorem 2.4] when $A \in \mathbb{C}_{n}^{\mathrm{CM}}$. In this case, we have $A=Q_{1} R_{1}, Q_{1}^{*} Q_{1}=I_{r}$ where $r$ the rank of $A$ and thus, the formula based on $\mathrm{QR}$ decomposition can also be simplified as

$$
A^{\oplus}=A^{\oplus}=Q_{1}\left(Q_{1}^{*} A Q_{1}\right)^{-1} Q_{1}^{*}=Q_{1}\left(Q_{1}^{*} Q_{1} R_{1} Q_{1}\right)^{-1} Q_{1}^{*}=Q_{1}\left(R_{1} Q_{1}\right)^{-1} Q_{1}^{*} .
$$

The novel expression (3.6) can be used to compute the core-EP inverse of matrices of an arbitrary index effectively if a subroutine for QR decomposition of a matrix is available on your computer. Many popular commercial software such MATLAB and MAPLE include such a package. For a large scale problem, that is the method of choice for a reliable solution as we know QR-based methods are always stable. But if the subroutine for QR decomposition is not available on your computer, then you may want to find an alternative method. The Gaussian elimination method that we are going to propose next is a good alternative if the problem you intend to solve is not too big since you can perform elementary operations by hands, not to mention the fact that many commercial scientific packages include a subroutine for computing the reduced row echelon form so you may utilize the subroutine to perform elementary row operations for you if needed.

The Gauss-Jordan method for the inverse of an invertible matrix $A$ is a method for computing the inverse of $A$ by performing elementary row operations on $[A \mid I]$ to reach the form of $[I \mid X]$. Then the inverse of $A$ can be read off from the second block of $[I \mid X]$. For a small scale problem, this method is very handy since it can be carried out easily by hands. This method has been recently adapted to compute various generalized inverses in the articles [1, 16-18, 24,35-37,39] and the references within these papers. The most recent one is for computing the core inverse of matrices of index one [37]. Due to the complexity of the generalized inverses, we are no longer able to start with the matrix $[A \mid I]$. The starting matrix depends on the explicit expression for that generalized inverse but the idea is the same - using elementary row or column operations on certain form of matrices to get all the building blocks of the explicit expression involved. For example, consider the explicit expression for $A^{\oplus}$ in Theorem 3.2

$$
A^{\oplus}=U_{k}\left(U_{k}^{*} A U_{k}\right)^{-1} U_{k}^{*}
$$


where $k=\operatorname{Ind}(A), U_{k} \in \mathbb{C}_{s}^{n \times s}$ with $\mathcal{R}\left(U_{k}\right)=\mathcal{R}\left(A^{k}\right)$ and $s=\operatorname{rank}\left(A^{k}\right)$. Our building blocks of this expression are the index $k$ and the matrix $U_{k}$ whose columns form a basis for $\mathcal{R}\left(A^{k}\right)$. Assume that $k$ is given (which can also be found through elementary operations). Then our task is to find such a matrix $U_{k}$. Since $\mathcal{R}\left(A^{k}\right)=$ $\mathcal{R}\left(A^{k} P\right)$ for any nonsingular matrix $P$, we can perform elementary column operations on $A^{k}$ without changing its column space. If we get $\left[U_{k} \mid O\right]$ with the property that the columns of $U_{k} \in \mathbb{C}^{n \times s}$ are linearly independent, then a basis for $\mathcal{R}\left(A^{k}\right)$ is found. The next step is to compute $U_{k}^{*} A U_{k}$, form

$$
\left(\begin{array}{cc}
U_{k}^{*} A U_{k} & U_{k}^{*} \\
U_{k} & O
\end{array}\right)
$$

and perform elementary row operations on the first $s$ rows of the matrix in (3.9) so that the block matrix (3.9) becomes

$$
\left(\begin{array}{cc}
I_{S} & \left(U_{k}^{*} A U_{k}\right)^{-1} U_{k}^{*} \\
U_{k} & O
\end{array}\right),
$$

where $I_{S}$ is the identity matrix of order $s$. Finally, performing elementary row operations on the block matrix (3.10) to get rid of the $(2,1)$-block of the block matrix (3.10), one will end up with

$$
\left(\begin{array}{cc}
I_{s} & \left(U_{k}^{*} A U_{k}\right)^{-1} U_{k}^{*} \\
O & -U_{k}\left(U_{k}^{*} A U_{k}\right)^{-1} U_{k}^{*}
\end{array}\right) .
$$

In this example, $-A^{\oplus}$ is the $(2,2)$-block of the block matrix (3.11). Therefore, we have successfully revealed the core-EP inverse $A^{\oplus}$ of $A$ using elementary row and column operations.

In summary, we have developed the Gaussian elimination method for computing the core-EP inverse of matrices of an arbitrary index.

We remark that Algorithm 1 reduces to Algorithm 3.1 in [37] when $A$ is of index one.

We end up this section with three examples of computing the core-EP inverse of a matrix in [30] using three different expressions in Theorems 3.1, 3.2, and Corollary 3.1. To save the space, we choose to discuss the matrix of order 3 but we have worked on a few randomly generated matrices of larger dimensions. All these three expressions performed very well on our limited randomly generated problems. Since this paper is focused more on the theoretical development, we omit to include the analysis of their numerical performance. 


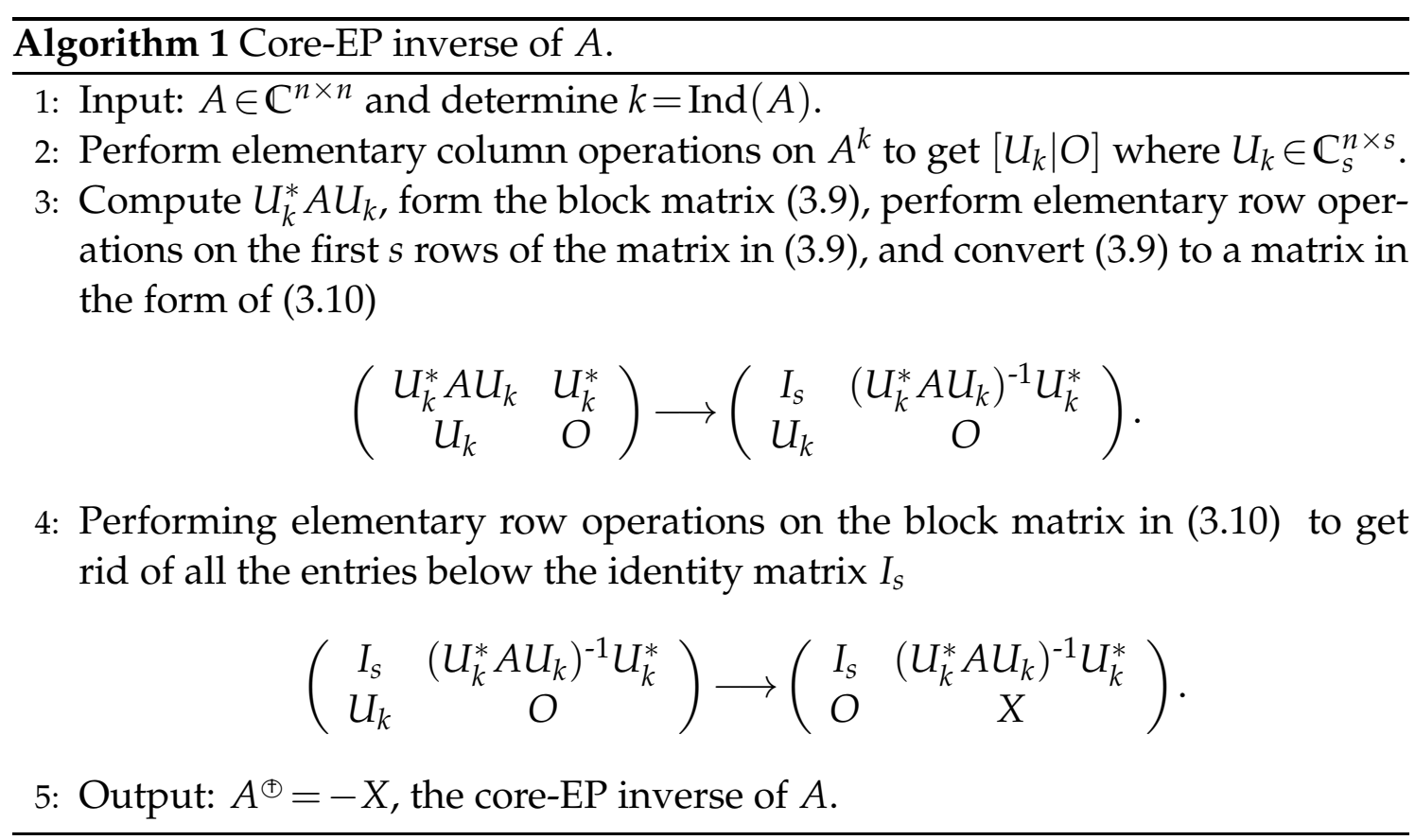

Example 3.1 ([30]). Using the Gaussian elimination method to compute the coreEP inverse $A^{\oplus}$ of the matrix $A$ where

$$
A=\left(\begin{array}{ccc}
1 & 1 & -1 \\
1 & 0 & 2 \\
2 & 1 & 1
\end{array}\right)
$$

For this matrix, $k=\operatorname{Ind}(A)=2$ and perform elementary column operations on $A^{2}$

$$
A^{2}=\left(\begin{array}{lll}
0 & 0 & 0 \\
5 & 3 & 1 \\
5 & 3 & 1
\end{array}\right) \longrightarrow\left(\begin{array}{lll}
0 & 0 & 0 \\
1 & 0 & 0 \\
1 & 0 & 0
\end{array}\right)
$$

leading to $s=1$, and $U_{2}^{T}=(0,1,1)$. Compute $U_{2}^{T} A U_{2}=4$ and form the matrix (3.9)

$$
\left(\begin{array}{cc}
U_{2}^{*} A U_{k} & U_{2}^{*} \\
U_{2} & O
\end{array}\right)=\left(\begin{array}{cccc}
4 & 0 & 1 & 1 \\
0 & 0 & 0 & 0 \\
1 & 0 & 0 & 0 \\
1 & 0 & 0 & 0
\end{array}\right)
$$

Then, convert the $(1,1)$-element to 1 by dividing 4 on the first row and afterwards, 
eliminate the $(3,1)$ - and $(4,1)$-elements, resulting in

$$
\left(\begin{array}{rrrr}
1 & 0 & \frac{1}{4} & \frac{1}{4} \\
0 & 0 & 0 & 0 \\
0 & 0 & -\frac{1}{4} & -\frac{1}{4} \\
0 & 0 & -\frac{1}{4} & -\frac{1}{4}
\end{array}\right) .
$$

We read off $A^{\oplus}$ from the previous matrix:

$$
A^{\oplus}=\left(\begin{array}{ccc}
0 & 0 & 0 \\
0 & \frac{1}{4} & \frac{1}{4} \\
0 & \frac{1}{4} & \frac{1}{4}
\end{array}\right) .
$$

Example 3.2. Using the QR-based explicit expression (3.6) to compute the core-EP inverse $A^{\oplus}$ of the matrix $A$ given in Example 3.1.

For this matrix, $k=\operatorname{Ind}(A)=2, s=\operatorname{rank}\left(A^{2}\right)=1$. We perform the QR decomposition of $A$ using MATLAB R2017b installed on Dell's laptop XPS 13 with 8 GB memory. If only 4 decimal places are displayed, then the MATLAB command $[Q, R]=\operatorname{qr}\left(A^{2}\right)$ results in

$$
Q=\left(\begin{array}{ccc}
0 & 1.0000 & 0.0000 \\
-0.7071 & 0.0000 & -0.7071 \\
-0.7071 & 0.0000 & 0.7071
\end{array}\right), \quad R=\left(\begin{array}{ccc}
-7.0711 & -4.2426 & -1.4142 \\
0 & 0.0000 & 0.0000 \\
0 & 0 & 0.0000
\end{array}\right)
$$

Taking the first column of $Q$, we have $Q_{1}=(0,-0.7071,-0.7071)^{T}$ and thus, the expression (3.6) gives

$$
A^{\oplus}=Q_{1}\left(Q_{1}^{*} A Q_{1}\right)^{-1} Q_{1}^{*}=\left(\begin{array}{ccc}
0 & 0 & 0 \\
0 & 0.2500 & 0.2500 \\
0 & 0.2500 & 0.2500
\end{array}\right) .
$$

Example 3.3. Using the explicit expression in Theorem 3.1 to compute the core-EP inverse $A^{\oplus}$ of the matrix $A$ given in Example 3.1.

Again, we carry out all the calculations using MATLAB R2017b installed on Dell's laptop XPS 13 with 8 GB memory and the Moore-Penrose inverse is obtained by using the MATLAB command pinv. For this matrix, $k=\operatorname{Ind}(A)=2$, we have $A^{2}$ already given in Example 3.1 and compute

$$
\left(A^{2}\right)^{\dagger}=\left(\begin{array}{ccc}
0 & 0.0714 & 0.0714 \\
0 & 0.0429 & 0.0429 \\
0 & 0.0143 & 0.0143
\end{array}\right), \quad A^{2}\left(A^{2}\right)^{\dagger}=\left(\begin{array}{ccc}
0 & 0 & 0 \\
0 & 0.5000 & 0.5000 \\
0 & 0.5000 & 0.5000
\end{array}\right)
$$


leading to

$$
A^{\oplus}=A^{2}\left(A^{2}\right)^{\dagger}\left[I+(A-I)\left(A^{2}\right)\left(A^{2}\right)^{\dagger}\right]^{-1}=\left(\begin{array}{ccc}
0 & 0 & 0 \\
0 & 0.2500 & 0.2500 \\
0 & 0.2500 & 0.2500
\end{array}\right) .
$$

\section{The Cramer's rules}

The core-EP inverse $A^{\oplus}$ of $A$ in Theorem 3.2 is expressed in terms of a basis for the column space of $A^{k}$. Next, we will express it in terms of a basis for the orthogonal complement of the column space, i.e., the nullspace of $\left(A^{k}\right)^{*}$.

Theorem 4.1. Let $A \in \mathbb{C}^{n \times n}$ and $k=\operatorname{Ind}(A)$. Let $G$ be a matrix whose columns form a basis for $\mathcal{N}\left(\left(A^{k}\right)^{*}\right)$. Then, we have

$$
A^{\oplus}=\left(A^{k}\left(A^{k}\right)^{*} A+G G^{*}\right)^{-1} A^{k}\left(A^{k}\right)^{*} .
$$

Proof. Let $s=\operatorname{rank}\left(A^{k}\right)$. First, we show that $A^{k}\left(A^{k}\right)^{*} A+G G^{*}$ is nonsingular. Assume that $\left(A^{k}\left(A^{k}\right)^{*} A+G G^{*}\right) x=0$. Then, we have

$$
\begin{aligned}
A^{k}\left(A^{k}\right)^{*} A x & =-G G^{*} x \in \mathcal{R}\left(A^{k}\right) \cap \mathcal{R}(G) \\
& =\mathcal{R}\left(A^{k}\right) \cap \mathcal{R}(G)=\mathcal{R}\left(A^{k}\right) \cap \mathcal{N}\left(\left(A^{k}\right)^{*}\right) \\
& =\mathcal{R}\left(A^{k}\right) \cap \mathcal{R}\left(A^{k}\right)^{\perp}=\{0\}
\end{aligned}
$$

implying that $A^{k}\left(A^{k}\right)^{*} A x=-G G^{*} x=0$ which further leads to $G^{*} x=0$ since $G$ is of full column rank. Now, we have

$$
x \in \mathcal{N}\left(G^{*}\right)=\mathcal{R}(G)^{\perp}=\mathcal{N}\left(\left(A^{k}\right)^{*}\right)^{\perp}=\mathcal{R}\left(A^{k}\right),
$$

leading to

$$
A x \in A \mathcal{R}\left(A^{k}\right)=\mathcal{R}\left(A^{k+1}\right)=\mathcal{R}\left(A^{k}\right) .
$$

On the other hand, it follows from $A^{k}\left(A^{k}\right)^{*} A x=0$ that

$$
A x \in \mathcal{N}\left(A^{k}\left(A^{k}\right)^{*}\right)=\mathcal{N}\left(\left(A^{k}\right)^{*}\right)=\mathcal{R}\left(A^{k}\right)^{\perp} .
$$

Therefore, we have

$$
A x \in \mathcal{R}\left(A^{k}\right) \cap \mathcal{R}\left(A^{k}\right)^{\perp}=\{0\}
$$


implying $A x=0$. That is, we have $x \in \mathcal{N}\left(A^{k}\right)$. Together with $x \in \mathcal{R}\left(A^{k}\right)$, we finally get

$$
x \in \mathcal{N}\left(A^{k}\right) \cap \mathcal{R}\left(A^{k}\right)=\{0\}
$$

and thus, $A^{k}\left(A^{k}\right)^{*} A+G G^{*}$ is nonsingular.

In view of (1.14), together with the fact that $G^{*} A^{k}=0$, we can write

$$
\begin{aligned}
\left(A^{k}\left(A^{k}\right)^{*} A+G G^{*}\right) A^{\oplus} & =\left(A^{k}\left(A^{k}\right)^{*} A+G G^{*}\right) A^{D} A^{k}\left(A^{k}\right)^{\dagger} \\
& =A^{k}\left(A^{k}\right)^{*} A A^{D} A^{k}\left(A^{k}\right)^{\dagger}+G G^{*} A^{D} A^{k}\left(A^{k}\right)^{\dagger} \\
& =A^{k}\left(A^{k}\right)^{*}\left(A^{k}\left(A^{k}\right)^{\dagger}\right)^{*}+G G^{*} A^{k} A^{D}\left(A^{k}\right)^{\dagger} \\
& =A^{k}\left(A^{k}\right)^{*}
\end{aligned}
$$

leading to the expression in the theorem since $A^{k}\left(A^{k}\right)^{*} A+G G^{*}$ is nonsingular.

Finally, let us turn our attention to an expression for the core-EP inverse using a bordered matrix.

Theorem 4.2. Let $A \in \mathbb{C}^{n \times n}, k=\operatorname{Ind}(A)$, and $s=\operatorname{rank}\left(A^{k}\right)$. Let $G$ be a matrix whose columns form a basis for $\mathcal{N}\left(\left(A^{k}\right)^{*}\right)$. Then, the bordered matrix

$$
\left(\begin{array}{cc}
A & G \\
G^{*} & O
\end{array}\right)
$$

is nonsingular and

$$
\left(\begin{array}{cc}
A & G \\
G^{*} & O
\end{array}\right)^{-1}=\left(\begin{array}{cc}
A^{\oplus} & \left(I-A^{\oplus} A\right) G\left(G^{*} G\right)^{-1} \\
\left(G^{*} G\right)^{-1} G^{*} & -\left(G^{*} G\right)^{-1} G^{*} A G\left(G^{*} G\right)^{-1}
\end{array}\right) .
$$

Proof. The result follows directly from [27, Theorem 2.3] by taking $B=G$ and $C=G^{*}$ since we have

$$
\mathcal{R}(B)=\mathcal{R}(G)=\mathcal{N}\left(\left(A^{k}\right)^{*}\right), \quad \mathcal{N}(C)=\mathcal{N}\left(G^{*}\right)=\mathcal{R}(G)^{\perp}=\mathcal{R}\left(A^{k}\right),
$$

and the fact that $G^{*} A^{k}=O$.

The core-EP inverse of the bordered matrix in Theorem 4.2 is also considered in [31]. We notice that the $(1,2)$ - and $(2,1)$-blocks of the regular inverse (4.3) of the bordered matrix in (4.2) are not explicitly specified in [31]. Of course, they 
are insignificant for the computation of the core-EP inverse using the bordered matrix since only the $(1,1)$-block of $(4.3)$ is relevant to $A^{\oplus}$.

The expressions in Theorems 4.1 and 4.2 can be used to obtain Cramer-like rules for computing the least squares solution to the constrained system of linear equations (1.7) and the core-EP inverse of $A$. For a survey of Cramer's rules for various generalized inverses, please refer to [45].

We first consider the Cramer's rules for the least squares solution to the constrained system of linear equations (1.7) and two determinantal expressions for the components of the core-EP inverse of $A$. To this end, let us first introduce a notation. Given a matrix $X \in \mathbb{C}^{n \times n}$ and a vector $c \in C^{n}$, when the $j$-th column of $X$ is replaced by $b$, the resulted matrix is denoted by $X(j \rightarrow c)$.

Theorem 4.3. Let $A \in \mathbb{C}^{n \times n}$ and $k=\operatorname{Ind}(A)$. Let $G$ be a matrix whose columns form a basis for $\mathcal{N}\left(\left(A^{k}\right)^{*}\right)$. If $b \notin \mathcal{R}\left(A^{k}\right)$, then the components of the least squares solution $x$ of (1.7) are

$$
x_{j}=\frac{\operatorname{det}\left(\left(A^{k}\left(A^{k}\right)^{*} A+G G^{*}\right)\left(j \rightarrow A^{k}\left(A^{k}\right)^{*} b\right)\right)}{\operatorname{det}\left(A^{k}\left(A^{k}\right)^{*} A+G G^{*}\right)}, j=1, \ldots, n .
$$

Proof. In view of Theorems 2.3 and 4.1, the least squares solution $x=A^{\oplus} b$ of the constrained system of linear equations (1.7) can be expressed as

$$
x=A^{\oplus} b=\left(A^{k}\left(A^{k}\right)^{*} A+G G^{*}\right)^{-1} A^{k}\left(A^{k}\right)^{*} b
$$

and it is the unique solution to the system of linear equations

$$
\left(A^{k}\left(A^{k}\right)^{*} A+G G^{*}\right) x=A^{k}\left(A^{k}\right)^{*} b .
$$

The result of the theorem follows immediately from the classic Cramer's rule applied to the system of linear equations (4.4).

Working with the bordered matrix in Theorem 4.2, we have the second Cramer's rule for the least squares solution to (1.7).

Theorem 4.4. Let $A \in \mathbb{C}^{n \times n}$ and $k=\operatorname{Ind}(A)$. Let $G$ be a matrix whose columns form a basis for $\mathcal{N}\left(\left(A^{k}\right)^{*}\right)$. If $b \notin \mathcal{R}\left(A^{k}\right)$, then the components of the least squares solution $x$ of (1.7) are

$$
x_{j}=\frac{\operatorname{det}\left(\begin{array}{cc}
A(j \rightarrow b) & G \\
G^{*}(j \rightarrow 0) & O
\end{array}\right)}{\operatorname{det}\left(\begin{array}{cc}
A & G \\
G^{*} & O
\end{array}\right)}, j=1, \ldots, n
$$


Proof. In view of Theorem 4.2, we have

$$
\left(\begin{array}{cc}
A & G \\
G^{*} & O
\end{array}\right)^{-1}\left(\begin{array}{l}
b \\
0
\end{array}\right)=\left(\begin{array}{c}
A^{\oplus} b \\
\left(G^{*} G\right)^{-1} G^{*} b
\end{array}\right) .
$$

Therefore, the least squares solution $x=A^{\oplus} b$ to the constrained system of linear equations (1.7) is the vector consisting of the first $n$ components of the solution of the following system of linear equations:

$$
\left(\begin{array}{cc}
A & G \\
G^{*} & O
\end{array}\right)\left(\begin{array}{l}
x \\
y
\end{array}\right)=\left(\begin{array}{l}
b \\
0
\end{array}\right)
$$

The result of the theorem follows immediately from the classic Cramer's rule applied to the system of linear equations (4.5).

Following the steps of $[14,15]$, we can easily establish two determinantal expressions for the core-EP inverse $A^{\oplus}$ since

$$
A_{i, j}^{\oplus}=e_{i}^{T}\left(A^{\oplus} e_{j}\right) .
$$

That is, $A_{i, j}^{\oplus}$ is the $i$ th component of $A^{\oplus} e_{j}$. The following theorem follows directly from Theorems 4.3 and 4.4 .

Theorem 4.5. Let $A \in \mathbb{C}^{n \times n}$ and $k=\operatorname{Ind}(A)$. Let $G$ be a matrix whose columns form a basis for $\mathcal{N}\left(\left(A^{k}\right)^{*}\right)$. Then, the components of the core-EP inverse $A^{\oplus}$ of $A$ are either from

$$
A_{i, j}^{\oplus}=\frac{\operatorname{det}\left(\left(A^{k}\left(A^{k}\right)^{*} A+G G^{*}\right)\left(i \rightarrow A^{k}\left(A^{k}\right)^{*} e_{j}\right)\right)}{\operatorname{det}\left(A^{k}\left(A^{k}\right)^{*} A+G G^{*}\right)}, \quad i, j=1, \ldots, n
$$

or

$$
A_{i, j}^{\oplus}=\frac{\operatorname{det}\left(\begin{array}{cc}
A\left(i \rightarrow e_{j}\right) & G \\
G^{*}(i \rightarrow 0) & O
\end{array}\right)}{\operatorname{det}\left(\begin{array}{cc}
A & G \\
G^{*} & O
\end{array}\right)}, \quad i, j=1, \ldots, n .
$$

Theorems 4.1, 4.3, 4.4, and (4.6) of Theorem 4.5 reduce to similar results in [46] for the core inverse of $A \in \mathbb{C}_{n}^{C M}$. Our results are for matrices of an arbitrary index. 


\section{The weighted core-EP inverse and a more general constrained system}

The core-EP inverse of a square matrix was recently extended to a rectangular matrix $A \in \mathbb{C}^{m \times n}$ by Ferreyra et al. [11]. Let $W \in \mathbb{C}^{n \times m}$ and $k=\max \{\operatorname{Ind}(A W)$, $\operatorname{Ind}(W A)\}$. The $W$-weighted core-EP inverse of $A$, denoted by $A^{\oplus, W}$, is the unique solution to the system

$$
W A W X=(W A)^{k}\left[(W A)^{k}\right]^{\dagger}, \quad \mathcal{R}(X) \subseteq \mathcal{R}(A W)^{k} .
$$

It is also the unique solution to the system [13, Theorems $2.2 \& 2.3$ ]

$$
X W(A W)^{k+1}=(A W)^{k}, \quad A W X W X=X, \quad(W A W X)^{*}=W A W X .
$$

The W-weighted core-EP inverse of $A$ can be computed through Moore-Penrose, core-EP, and $\mathrm{W}$-weighted Drazin inverses of $A$ as indicated by the following explicit expressions

$$
\begin{aligned}
& A^{\oplus, W}=\left(W(A W)^{l+1}\left[(A W)^{l}\right]^{\dagger}\right)^{\dagger}, \\
& A^{\oplus, W}=A\left[(W A)^{\oplus}\right]^{2}, \\
& A^{\oplus, W}=(A W)^{l}\left[W(A W)^{l+1}\right]^{\dagger}, \\
& A^{\oplus, W}=A^{D, W} P_{\mathcal{R}\left((W A)^{k}\right),}
\end{aligned}
$$

where $l \geq k$ and $A^{D, W}$ is the $\mathrm{W}$-weighted Drazin inverse of $A$ defined in [10]. More explicit expressions and interesting properties for the weighted core-EP inverse can be found in $[11,13,22]$.

In this section, we show that the $\mathrm{W}$-weighted core-EP inverse of $A$ is closely related to the solution of the following constrained system of linear equations

$$
\min _{x \in \mathcal{R}\left((A W)^{k}\right)}\|W A W x-b\|_{2}
$$

where $A \in \mathbb{C}^{m \times n}, W \in \mathbb{C}^{n \times m}, b \in C^{n}$ and $k=\max \{\operatorname{Ind}(A W)$, $\operatorname{Ind}(W A)\}$. This problem is an extension of (1.7) since (5.3) reduces to (1.7) for the case when $m=n$ and $W=$ I. Obviously, the least squares problem (5.3) contains the consistent constrained system $W A W x=b, x \in \mathcal{R}\left((A W)^{k}\right)$ in which the minimum value of the least squares problem (5.3) is zero.

Lemma 5.1. Let $A \in \mathbb{C}^{m \times n}, W \in \mathbb{C}^{n \times m}, b \in \mathbb{C}^{n}$, and $l \geq k=\operatorname{Ind}(W A)$. Then we have $\mathcal{R}\left(W(A W)^{l+1}\right)=\mathcal{R}\left((W A)^{k}\right)$. 
Proof. We can write

$$
\begin{aligned}
\mathcal{R}\left(W(A W)^{l+1}\right) & =\mathcal{R}\left((W A)^{l+1} W\right) \subseteq \mathcal{R}\left((W A)^{l+1}\right)=\mathcal{R}\left((W A)^{l+2}\right) \\
& =\mathcal{R}\left(W(A W)^{l+1} A\right) \subseteq \mathcal{R}\left(W(A W)^{l+1}\right)
\end{aligned}
$$

implying that

$$
\mathcal{R}\left(W(A W)^{l+1}\right)=\mathcal{R}\left((W A)^{l+1}\right)=\mathcal{R}\left((W A)^{k}\right) .
$$

Theorem 5.1. Let $A \in \mathbb{C}^{m \times n}, W \in \mathbb{C}^{n \times m}, b \in \mathbb{C}^{n}$, and $l \geq k=\max \{\operatorname{Ind}(A W)$, $\operatorname{Ind}(W A)\}$. Then, $(A W)^{l} Y$ is the $W$-weighted core-EP inverse of $A$ if and only if $Y$ is a $\{1,3\}$-inverse of $W(A W)^{l+1}$.

Proof. IF: Let

$$
X=(A W)^{l}\left(W(A W)^{l+1}\right)^{(1,3)}
$$

Obviously, we have

$$
\mathcal{R}(X) \subseteq \mathcal{R}\left((A W)^{l}\right)=\mathcal{R}\left((A W)^{k}\right)
$$

and

$$
\begin{aligned}
W A W X & =W A W(A W)^{l}\left(W(A W)^{l+1}\right)^{(1,3)} \\
& =W(A W)^{l+1}\left(W(A W)^{l+1}\right)^{(1,3)} \\
& =P_{\mathcal{R}\left(W(A W)^{l+1}\right)}=P_{\mathcal{R}\left((W A)^{k}\right)} \\
& =(W A)^{k}\left[(W A)^{k}\right]^{\dagger}
\end{aligned}
$$

Thus, in view of (5.1), $X$ is the $W$-weighted core-EP inverse of $A$, i.e.,

$$
A^{\oplus, W}=(A W)^{l}\left(W(A W)^{l+1}\right)^{(1,3)} .
$$

ONLY IF: Assume that $(A W)^{l} Y=A^{\oplus, W}$. It follows from (5.1) that

$$
\begin{aligned}
W(A W)^{l+1} Y & =W(A W)(A W)^{l} Y=W(A W) A^{\oplus, W} \\
& =(W A)^{k}\left[(W A)^{k}\right]^{\dagger}
\end{aligned}
$$

leading to

$$
\left(W(A W)^{l+1} \Upsilon\right)^{*}=W(A W)^{l+1} \Upsilon
$$


Moreover, in view of (5.6) and Lemma 5.1, we can write $W(A W)^{l+1}=(W A)^{k} Z$ for some $Z$ and

$$
\begin{aligned}
W(A W)^{l+1} Y W(A W)^{l+1} & =(W A)^{k}\left[(W A)^{k}\right]^{\dagger}(W A)^{k} Z \\
& =(W A)^{k} Z=W(A W)^{l+1}
\end{aligned}
$$

Therefore, $Y$ is a $\{1,3\}$-inverse of $W(A W)^{l+1}$.

Now, let us return to (5.3). To remove the constraint, we set $x=(A W)^{k} y$ and thus, we have

$$
\min _{x \in \mathcal{R}\left((A W)^{k}\right)}\|W A W x-b\|_{2}=\min _{y \in \mathbb{C}^{m}}\left\|W(A W)^{k+1} y-b\right\|_{2^{\prime}}
$$

whose general solution is

$$
y=\left(W(A W)^{k+1}\right)^{(1,3)} b+\left(I-\left(W(A W)^{k+1}\right)^{(1,3)} W(A W)^{k+1}\right) q
$$

for an arbitrary $q$. Thus, in view of (5.5), the general solution to (5.3) is

$$
\begin{aligned}
x= & (A W)^{k} y=(A W)^{k}\left(W(A W)^{k+1}\right)^{(1,3)} b \\
& +(A W)^{k}\left(I-\left(W(A W)^{k+1}\right)^{(1,3)} W(A W)^{k+1}\right) q \\
= & A^{\oplus, W} b+p,
\end{aligned}
$$

where

$$
p=(A W)^{k}\left(I-\left(W(A W)^{k+1}\right)^{(1,3)} W(A W)^{k+1}\right) q .
$$

In view of Lemma 5.1 and (5.4), we have

$$
\begin{aligned}
W A W p & =W(A W)^{k+1}\left(I-\left(W(A W)^{k+1}\right)^{(1,3)} W(A W)^{k+1}\right) q \\
& =\left(I-W(A W)^{k+1}\left(W(A W)^{k+1}\right)^{(1,3)}\right) W(A W)^{k+1} q \\
& =\left(I-(W A)^{k}\left[(W A)^{k}\right]^{\dagger}\right) W(A W)^{k+1} q=0
\end{aligned}
$$

implying that

$$
A W p \in \mathcal{N}(W) \subseteq \mathcal{N}(A W) \subseteq \mathcal{N}\left((A W)^{k}\right)
$$


In addition, $A W p \in \mathcal{R}\left((A W)^{k}\right)$ so $A W p=0$. Thus, $p \in \mathcal{N}(A W) \subseteq \mathcal{N}\left((A W)^{k}\right)$ which, together with the fact that $p \in \mathcal{R}\left((A W)^{k}\right)$, further implies that $p=0$. Therefore, in view of (5.7), we have a unique solution $x=A^{\oplus, \mathrm{W}} b$ to (5.3).

In summary, we have proved the following result.

Theorem 5.2. Let $A \in \mathbb{C}^{m \times n}, W \in \mathbb{C}^{n \times m}, b \in C^{n}$, and $l \geq k=\max \{\operatorname{Ind}(A W)$, $\operatorname{Ind}(W A)\}$. Then, the constrained system (5.3) has a unique solution $A^{\oplus, W_{b}}$.

Define $z=b-W A W x$. In view of (5.4), (5.5), (5.7), and (5.8), we have

$$
z=\left(I-W A W A^{\oplus, W}\right) b=\left(I-P_{\mathcal{R}\left((W A)^{k}\right)}\right) b=P_{\mathcal{R}\left((W A)^{k}\right)^{\perp}} b .
$$

Thus, for any $b$, there exist unique $x=A^{\oplus, W} b \in \mathcal{R}\left((A W)^{k}\right)$ and $z=P_{\mathcal{R}\left((W A)^{k}\right)^{\perp}} b \in$ $\mathcal{R}\left((W A)^{k}\right)^{\perp}$ such that $b=W A W x+z$. But in general, $\mathcal{R}\left((W A)^{k}\right) \neq \mathcal{R}\left((A W)^{k}\right)$ for a general $W$ and thus, $A^{\oplus, W}$ may not be the Bott-Duffin inverse of $W A W$ with respect to $\mathcal{R}\left((A W)^{k}\right)$ for a general $W$. Since the beautiful expression in Theorem 3.2 for the core-EP inverse is based on the fact that the core-EP inverse of a square matrix $A$ is the Bott-Duffin inverse of $A$ with respect to $\mathcal{R}\left(A^{k}\right)$, we do not have similar simple expressions for the $W$-weighted core-EP inverse of a rectangular matrix. At this junction, it is worthy of mentioning two expressions in that nature for $A^{\oplus, W}$, using the full-rank decomposition of $A(W A)^{k}\left[(W A)^{k}\right]^{\dagger}[13$, Corollary 2.7] and $(W A)^{k}[22$, Theorem 2.3], respectively.

\section{Acknowledgments}

We would like to thank the editor and three reviewers for their careful reading of the manuscript and useful suggestions.

\section{References}

[1] K. M. Anstreicher and U. G. Rothblum, Using Gauss-Jordan elimination to compute the index, generalized null spaces and Drazin inverse, Linear Algebra Appl. 85 (1987), 221-239.

[2] O. M. Baksalary and G. Trenkler, Core inverse of matrices, Linear Multilinear Algebra 58 (2010), 681-697.

[3] O. M. Baksalary and G. Trenkler, On a generalized core inverse, Appl. Math. Comput. 236 (2014), 450-457.

[4] A. Ben-Israel, A Cramer rule for least-norm solutions of consistent linear equations, Linear Algebra Appl. 43 (1982), 223-226. 
[5] A. Ben-Israel and T. N. E. Greville, Generalized Inverse: Theory and Applications, John Wiley, 2003.

[6] R. Bott and R. J. Duffin, On the algebra of networks, Trans. Amer. Math. Soc. 74 (1953), 99-109.

[7] S. L. Campbell and C. D. Meyer, Generalized Inverses of Linear Transformations, SIAM, 2009.

[8] Y. L. Chen, Representations and Cramer rules for the solution of a restricted matrix equation, Linear Multilinear Algebra 35 (1993), 339-354.

[9] Y. L. Chen, Expressions and determinantal formulas for the generalized inverse $A_{T, S}^{(2)}$ and their applications, J. Najing Normal University (Natural Science) 14 (1993) no. 4, 3-14.

[10] R. E. Cline and T. N. E. Greville, A Drazin inverse for rectangular matrices, Linear Algebra Appl. 29 (1980), 53-62.

[11] D. E. Ferreyra, F. E. Levis, and N. Thome, Revisiting the core EP inverse and its extension to rectangular matrices, Quaest. Math. 41 (2018), 265-281.

[12] Y. Gao and J. Chen, Pseudo core inverses in rings with involution, Comm. Algebra 46 (2018), 38-50.

[13] Y. Gao, J. Chen, and P. Patricio, Representations and properties of the weighted core-EP inverse, Linear Multilinear Algebra, 2018, DOI: 10.1080/03081087.2018.1535573.

[14] J. Ji, Explicit expressions of the generalized inverses and condensed Cramer rules, Linear Algebra Appl. 404 (2005), 183-192.

[15] J. Ji, A condensed Cramer's rule for the minimum-norm least-squares solution of linear equations, Linear Algebra Appl. 437 (2012), 2173-2178.

[16] J. Ji, Gauss-Jordan elimination methods for the Moore-Penrose inverse of a matrix, Linear Algebra Appl. 437 (2012), 1835-1844.

[17] J. Ji, Two inverse-of-N-free methods for $A_{M, N}^{+}$, Appl. Math. Comput. 232 (2014), 39-48.

[18] J. Ji and X. Z. Chen, A new method for computing Moore-Penrose inverse through GaussJordan elimination, Appl. Math. Comput. 245 (2014), 271-278.

[19] J. Ji and Y. Wei, The Drazin inverse of an even-order tensor and its application to singular tensor equations, Comput. Math. Appl. 75 (2018), 3402-3413.

[20] I. Kyrchei, Determinantal representations of the core inverse and its generalizations with applications, J. Math. 2019 (2019), 1-13.

[21] H. Ma, Optimal perturbation bounds for the core inverse, Appl. Math. Comput. 336 (2018), 176-181.

[22] H. Ma, A characterization and perturbation bounds for the weighted core-EP inverse, Quaestiones Mathematicae 43 (2020), 869-879.

[23] H. Ma, Displacement structure of the core inverse, Linear Multilinear Algebra 2020, https://doi.org/10.1080/03081087.2020.1716677.

[24] J. Ma, F. Gao, and Y. Li, An efficient method for computing the outer inverse $A_{T, S}^{(2)}$ through Gauss-Jordan elimination, Numer. Algorithms 85 (2020), 77-106.

[25] H. Ma, X. Gao and P. S. Stanimirovic, Characterizations, iterative method, sign pattern 
and perturbation analysis for the DMP inverse with its applications, Appl. Math. Comput. 378 (2020), DOI: 10.1016/j.amc.2020.125196.

[26] H. Ma and T. T. Li, Characterizations and representations of the core inverse and its applications, Linear Multilinear Algebra (2019), DOI: 10.1080/03081087.2019.1588847.

[27] H. Ma and P. S. Stanimirovic, Characterizations, approximation and perturbations of the core-EP inverse Characterizations, approximation and perturbations of the core-EP inverse, Appl. Math. Comput. 359 (2019), 404-417.

[28] S. B. Malik, L. Rueda, and N. Thome, Further properties on the core partial order and other matrix partial orders, Linear Multilinear Algebra 62 (2014), 1629-1648.

[29] S. B. Malik and N. Thome, On a new generalized inverse for matrices of an arbitrary index, Appl. Math. Comput. 226 (2014), 575-580.

[30] K. M. Prasad and K. S. Mohana, Core-EP inverse, Linear Multilinear Algebra 62 (2014), 792-802.

[31] K. M. Prasad and M. D. Raj, Bordering method to compute core-EP inverse, Spec. Matrices 6 (2018), 193-200.

[32] K. M. Prasad, M. D. Raj, M. Vinay, Iterative method to find core-EP inverse, Bull. Kerala Math. Assoc., Special Issue 16 (2018), 139-152.

[33] D. S. Rakic, N. C. Dincic and D. S. Djordjevic, Core inverse and core partial order of Hilbert space operators, Appl. Math. Comput. 244 (2014), 283-302.

[34] X. P. Sheng, Computation of weighted Moore-Penrose inverse through Gauss-Jordan elimination on bordered matrices, Appl. Math. Comput. 323 (2018), 64-74.

[35] X. P. Sheng and G. L. Chen, A note of computation for M-P inverse $A^{\dagger}$, Int. J. Comput. Math. 87 (2010), 2235-2241.

[36] X. P. Sheng and G. L Chen, Innovation based on Gaussian elimination to compute generalized inverse $A_{T, S}^{(2)}$, Comput. Math. Appl. 65 (2013), 1823-1829.

[37] X. P. Sheng and D. Xin, Methods of Gauss-Jordan elimination to compute core inverse $A^{\oplus}$ and dual core inverse $A_{\oplus}$, Linear Multilinear Algebra July 2020, DOI:10.1080/ 03081087.2020.1796906.

[38] P. S. Stanimirovic, D. Pappas, V. N. Katsikis, and I. Stanimirovic, Full-rank representations of outer inverses based on the QR decomposition, Appl. Math. Comput. 218 (2012), 10321-10333.

[39] P. S. Stanimirovic and M. D. Petkovic, Gauss-Jordan elimination method for computing outer inverses, Appl. Math. Comput. 219 (2013), 4667-4679.

[40] H. X. Wang, Core-EP decomposition and its applications, Linear Algebra Appl. 508 (2016), 289-300.

[41] H. X. Wang and J. L. Chen, Weak group inverse, Open Math. 16 (2018), 1218-1232.

[42] H. X. Wang, J. L. Chen, and G. Yan, Generalized Cayley-Hamilton theorem for core-EP inverse matrix and DMP inverse matrix, J. Southeast University (Engl. Ed.) 1 (2018), 135-138.

[43] H. X. Wang and X. J. Liu, Characterizations of the core inverse and the core partial order- 
ing, Linear Multilinear Algebra 63 (2015), 1829-1836.

[44] H. X. Wang and X. J. Liu, A partial order on the set of complex matrices with index one, Linear Multilinear Algebra 66 (2018), 206-216.

[45] G. Wang, Y. Wei, and S. Qiao, Generalized Inverses: Theory and Computations, Springer and Science Press, 2018.

[46] H. Wang and X. Zhang, The core inverse and constrained matrix approximation problem, Open Math. 18 (2020), 653-661.

[47] M. M. Zhou, J. L. Chen, T. T. Li, and D. G. Wang, Three limit representations of the core-EP inverse, Filomat 32 (2018), 5887-5894.

[48] M. Zhou, J. Chen, P. Stanimirovic, V. Katsikis, and H. Ma, Complex varying-parameter Zhang neural networks for computing core and core-EP inverse, Neural Process Lett. 51 (2020), 1299-1329. 